Submitted to

Plasma Sources Science and Technology

Invited Review

for $20^{\text {th }}$ Anniversary Edition, 2012

\title{
The evolution of ion charge states in cathodic vacuum arc plasmas: a review
}

\author{
André Anders* \\ Lawrence Berkeley National Laboratory, Berkeley, California \\ *email: aanders@lbl.gov
}

Revised version of December 18, 2011

\section{ACKNOWLEDGMENT}

Many of the here-collected results are based on ideas and contributions by close friends and colleagues. Running the risk of offending those not mentioned, I gratefully acknowledge the input over the years from just a few: B. Jüttner, E. Hantzsche, P. Siemroth, T. Schülke, I. Brown, E. Oks and G. Yushkov. This work was supported by the U.S. Department of Energy under Contract No. DE-AC02-05CH11231.

\section{DISCLAIMER}

This document was prepared as an account of work sponsored by the United States Government. While this document is believed to contain correct information, neither the United States Government nor any agency thereof, nor The Regents of the University of California, nor any of their employees, makes any warranty, express or implied, or assumes any legal responsibility for the accuracy, completeness, or usefulness of any information, apparatus, product, or process disclosed, or represents that its use would not infringe privately owned rights. Reference herein to any specific commercial product, process, or service by its trade name, trademark, manufacturer, or otherwise, does not necessarily constitute or imply its endorsement, recommendation, or favoring by the United States Government or any agency thereof, or The Regents of the University of California. The views and opinions of authors expressed herein do not necessarily state or reflect those of the United States Government or any agency thereof or The Regents of the University of California. 


\title{
The evolution of ion charge states in cathodic vacuum arc plasmas: a review
}

\section{André Anders}

Lawrence Berkeley National Laboratory, 1 Cyclotron Road, MS 53, Berkeley, California 94720, USA

Email: aanders@lbl.gov

\begin{abstract}
Cathodic vacuum arc plasmas are known to contain multiply charged ions. 20 years after "Pressure Ionization: its role in metal vapour vacuum arc plasmas and ion sources" appeared in vol. 1 of Plasma Sources Science and Technology, it is a great opportunity to re-visit the issue of pressure ionization, a non-ideal plasma effect, and put it in perspective to the many other factors that influence observable charge state distributions, such as the role of the cathode material, the path in the density-temperature phase diagram, the "noise" in vacuum arc plasma as described by a fractal model approach, the effects of external magnetic fields and charge exchange collisions with neutrals. A much more complex image of the vacuum arc plasma emerges putting decades of experimentation and modeling in perspective.
\end{abstract}

(Some figures in this article are in colour only in the electronic version) 


\section{Introduction}

Cathodic vacuum arc plasmas are among the most mysterious, difficult to understand, and uncontrolled plasma generators. Cathodic vacuum arc discharges are characterized by the formation of very small, non-stationary cathode spots - locations of extreme current and power densities - while the voltage between anode and cathode is relatively low $(\sim 20 \mathrm{~V})$ and fluctuating. The plasma made at the spots stems from the cathode material, i.e. the cathode is the feedstock and process gas is not needed, hence the name cathodic vacuum arc. For many practical applications, especially when depositing thin films and coatings, reactive gas is added. For example, to make a TiN coating one would use a titanium cathode and operate the discharge in a nitrogen-containing gas. In this case, the name cathodic arc is preferred, emphasizing the point that the cathode is the source of the metal plasma. In that sense, cathodic vacuum arcs a subcategory of cathodic arcs.

The most physical definition of an arc can be related to the discharge's mechanism of electron emission [1]. Arcs involve collective electron emission processes, such as thermionic emission [2], field emission [3], the non-linear combination of thermionic and field emission (thermofield emission) [4], and explosive electron emission [5], all of which are discussed in [6]. Collective mechanisms can be contrasted with individual mechanisms such as secondary electron emission upon ion impact on a cathode surface, which is typical for glow and magnetron discharges.

Among arcs, we distinguish thermionic arcs, based on thermionic electron emission from a hot cathode, and cathodic arcs, which are at least partially based on explosive emission. In this contribution, a review of our understanding of plasma formation of cathodic vacuum arcs is presented, taking the $20^{\text {th }}$ anniversary of the paper "Pressure ionization: its role in metal vapour vacuum arc plasmas and ion sources” [7] in volume 1 of Plasma Sources Science and Technology as the occasion. Even as this work has review character, no claim of being comprehensive is made. Rather, the report is largely based on research at Lawrence Berkeley National Laboratory with focus on clarifying the mechanisms that establish the observable ion charge state distributions, which are known to involve multiple charge states and depend of the cathode material.

\section{Path of cathode material in the phase diagram.}

One of the main points of the original paper [7] was an exploration how the solid cathodic material ends up as a fully ionized, expanding plasma far from the cathode body. The related heavy particle - temperature phase diagram is reproduced here as Fig. 1. The starting point of the journey is always the solid as indicated by the dot near the top left. We consider a microscopically small volume element of material at the cathode surface that exhibits stronger field emission than other locations. This can be caused by some form of surface irregularity or by the plasma itself. For example, the surface location may have a special feature there like a grain boundary, an edge, a protrusion, a scratch, or it is chemically modified due the formation of an oxide, hydroxide, nitride, etc., or a dust particle is present. Additionally, the plasma may be non-uniform and therefore especially dense at some location due to a previously occurring plasma formation event, or temporally enhanced due to a plasma fluctuation or instability. Locally dense plasma will provide intense heating of the surface due to positive ions being accelerated in the sheath toward the surface. The electric surface field can be enhanced simply because the sheath next to dense plasma is thinner than the sheath of less dense plasma. In all of those cases, the surface is locally non-uniformly heated by ion bombardment (bringing kinetic and potential energy to the surface), and Ohmic heating by the electron emission current. This can lead to a positive feedback: the more electrons are emitted the greater is the heating, which in turn can lead to a nonlinear amplification of the emission. Field emission turns into thermofield emission and then into explosive emission, where the material of the cathode volume under consideration explodes. The whole process can occur on a 
timescale of nanoseconds. This thermal runaway description is at the heart of the explosive emission models. The phase diagram (Fig. 1) considers the paths of the material, which can be quite different depending on when atoms are released within in the explosive process.

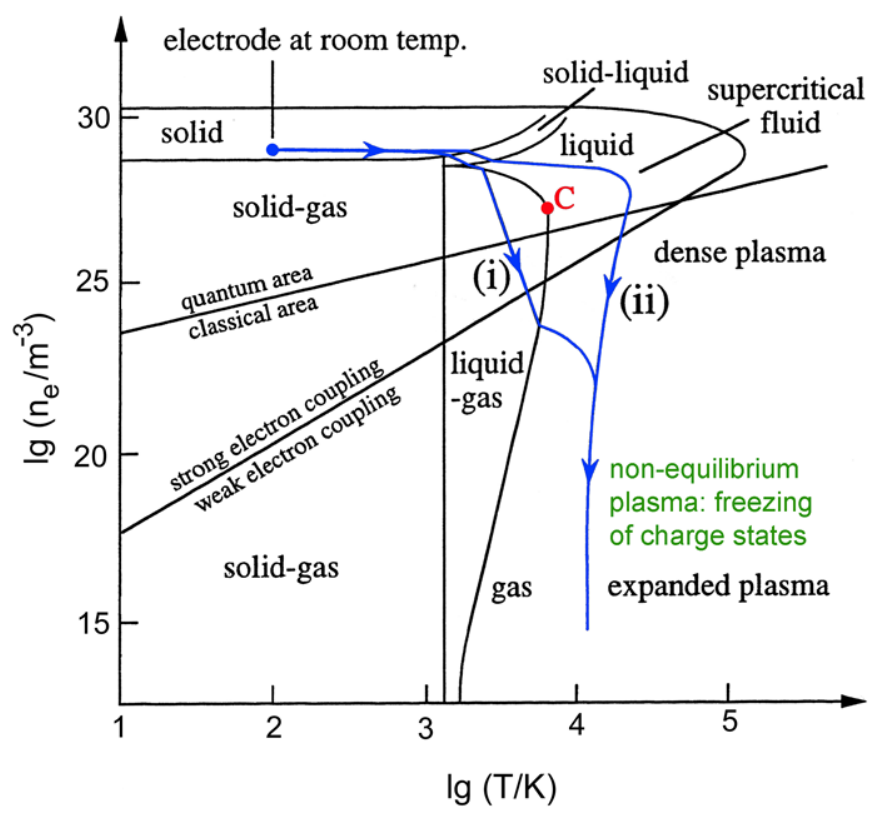

Figure 1. Paths of the cathode material in the heavy density-temperature phase diagram. Initially, the material is solid at room temperature (starting point in the upper left region of the diagram). The actual path strongly depends on the time relative to the "life stage" of the emitting site. In the initial explosive phase, the material may be heated beyond the critical point and transiently forms a dense, nonideal plasma. Later, more conventional melting and evaporation is dominant (after [7]).

Early in the fast runaway process, the density is not much changed due to inertia, and therefore the path appears horizontal, and the material may be heated beyond the solid-liquid phase transition circumnavigating the critical point "C". By definition, beyond "C" one cannot distinguish between liquid metal and dense, non-ideal plasma. As the atomic lattice bonds are destroyed by the strong thermal motion of the ions (i.e. the previous lattice atoms of the solid), a very high pressure can be assigned to this state of matter, and the pressure gradient due to the vacuum ambient causes a very rapid expansion. This is evident in the phase diagram by the sharp turn of the path from horizontal to vertical, going from heating to expansion and cooling.

As the explosive process evolves, the area of intense electron emission increases due to heat conduction. This leads to a decrease of the power density assuming that the current per emission site is limited and not increasing proportionally with the area (an assumption based on observations that higher discharge currents lead to a greater number of simultaneously operating emission sites [8, 9]). An increased emission area leads to a modified path characterized by a smaller rate of heating. The cathode material goes now through the more familiar phase transitions solid $\rightarrow$ liquid $\rightarrow$ gas $\rightarrow$ plasma, as indicated by the second, lower-density path. Those path representations are just two: the actual path changes as the spot development progresses. For example, late in a spot's "lifetime," only a hot crater is left on the surface, and this area does not produce plasma anymore but, depending on the cathode material and its vapor pressure, the crater may emit neutral metal vapor. This would be yet another path which is not shown in Fig. 1. 
Once the arc discharge is completed the cathode is covered with numerous craters: remnant evidence for the sequence of microexplosions and the locally extreme power density that existed at some stage in the emission site's evolution. As one can see from Fig. 2, similar craters of very different size exists, already suggesting that crater formation cannot be explained by a sequence of equal, short-lived elementary events, rather, a model spanning orders of magnitude is needed. The fractal model of section 4 can exactly do that.

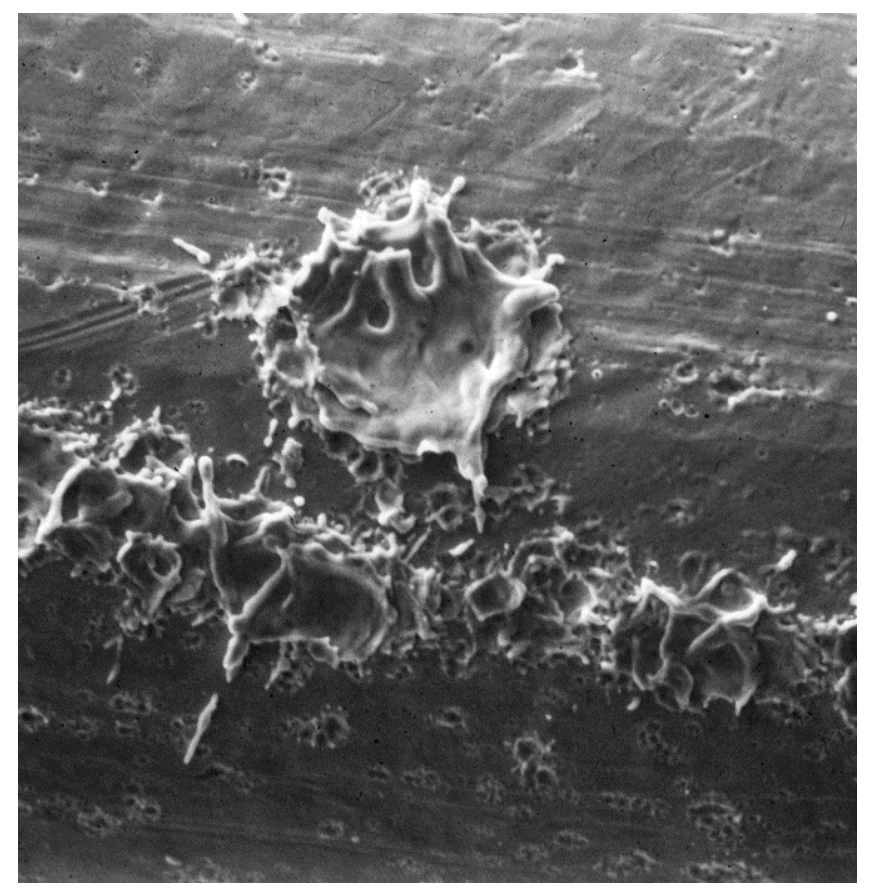

Figure 2. A variety of craters formed by a pulsed vacuum arc discharge on molybdenum in ultra-high vacuum (UHV). The molybdenum cathode was pre-cleaned by heating in UHV prior to operating the arc; a magnetic field caused the spot motion to be steered (as opposed to being random). Photo courtesy of B. Jüttner, Berlin.

\section{The Freezing model and its limitations. Nonideal plasma effects of pressure ionization.}

The theme of the original paper [7] was to consider what, if any, effect the strong interaction of particles has as the plasma goes through its dense phase near or beyond the critical point. The strong interaction or coupling between particles shifts the atomic energy level and thereby reduces the energy needed to ionize atoms and to produce multiply charged ions,

$$
E_{Q}^{e f f}=E_{Q}-\Delta E_{Q}
$$

where $E_{Q}$ is the energy needed to ionize (an isolated) ion of charge state $Q$ (with $Q=0$ being a neutral atom, $Q=1$ the singly charged ion, etc.), and $\Delta E_{Q}$ is the corresponding reduction due to many-particle interaction. The exact formulation was given in [7] and there is no need to reproduce the relatively elaborate scheme of equations here. It should be mentioned, though, that in the limit of a weakly nonideal plasma, the rather simple Debye-Hückel approximation 


$$
\Delta E_{Q}=\frac{(Q+1) e^{2}}{4 \pi \varepsilon_{0} \lambda_{D}}
$$

can be applied, where the Debye length (screening length) is given by

$$
\lambda_{D}=\left(\frac{\varepsilon_{0} k T}{e^{2}\left(n_{e}+\left\langle Q^{2}\right\rangle n_{i}\right)}\right)^{1 / 2}
$$

where \langle\rangle indicates averaging over the charge states; $e$ is the elementary charge, $\varepsilon_{0}$ is the permittivity of free space, $k$ is the Boltzmann constant, $T$ is the temperature, and $n_{e}$ and $n_{i}$ are the electron and ion density, respectively.

As the dense plasma expands from the emission site its local density and temperature decrease. As a result, the frequency of collisions between particles drops. Now it is useful to compare the rate of change for ion charge states due to the expansion versus that due to ionization and recombination [10]. In order to do that one can take a concept from chemical engineering and consider ionization and recombination like chemical reactions and introduce the Damköhler number $\mathscr{D} \boldsymbol{a}$ as a ratio of rates or as a ratio of characteristic times, as follows:

$$
\mathscr{D} \boldsymbol{a}=\frac{\text { reaction rate }}{\text { expansion rate }} \text { or } \mathfrak{D} \boldsymbol{a}=\frac{\text { characteristic expansion time }}{\text { characteristic reaction time }} \text {. }
$$

If $\mathscr{D} \boldsymbol{a} \ll 1$ the ionization and recombination processes can be neglected and the charge states are said to be frozen, i.e. the ion charge distribution function is not changing during the expansion (Fig. 3). In the opposite case, $\mathfrak{D} \boldsymbol{a} \gg 1$, the charge state distribution is in equilibrium for the local temperature and particle densities, and one can use the well-known Saha equations to express the ratios of charge states:

$$
S_{Q} \equiv \frac{n_{e} n_{Q+1}}{n_{Q}}=\Lambda_{B}^{-3} \frac{2 \Sigma_{Q+1}(T)}{\Sigma_{Q}(T)} \exp \left(-\frac{E_{Q}-\Delta E_{Q}}{k T}\right)
$$

where

$$
\Sigma_{Q}(T)=\sum_{s=1}^{s=s_{\max }} g_{Q, s} \exp \left(-\frac{E_{Q, s}}{k T}\right)
$$

are the partition functions which depend on the energy levels $E_{Q, s}$ of the ion and the level's statistical weights $g_{Q, s}$. In this way, the atomic structure of the ions constituting the plasma is taken into account. In equation (5), $\Lambda_{B}$ is called the thermal de Broglie length, defined as

$$
\Lambda_{B}=h /\left(2 \pi m_{e} k T\right)^{1 / 2},
$$

where $h$ is the Planck constant. The system is closed by the condition of quasi-neutrality, where the density of electrons (negative charge) is equal to density of all positive charges: 


$$
n_{e}=\sum_{Q}^{Q_{\max }} Q n_{Q}
$$

The characteristic expansion time can be defined as the time needed for the density of heavy particles (atoms and ions) to drop to half of its initial value (note: the sum of all atoms and ions is here used, since it is not affected by ionization and recombination reactions). Hence the expansion time can be written as [10]

$$
\tau_{\exp }(r)=\frac{\sqrt{2}-1}{v_{p l}} \sqrt{\gamma / n_{p l}(r)}
$$

where $v_{p l}$ is the plasmas expansion velocity (typically $10^{4} \mathrm{~m} / \mathrm{s}[11]$ ), and

$$
n_{p l} \approx \gamma \frac{I_{a r c}}{r^{2}}
$$

is the local plasma density at the distance $r$ from the emission site [12], and $\gamma$ is a constant (about $2 \times 10^{13} \mathrm{~A}^{-1} \mathrm{~m}^{-1}$ ). This relation is valid for low-current arcs where we can assume that the arc current is concentrated in a one emission site (generally less than $100 \mathrm{~A}$ ). In order to derive the characteristic times for ionization and recombination we need to solve the balance equations ( $c f$. chapter 4 of [6]):

$$
\begin{gathered}
\frac{\partial n_{Q}}{\partial t}=n_{Q+1} n_{e}^{2} \alpha_{Q+1, Q}-n_{Q} n_{e} \beta_{Q, Q+1} \text { for } Q=0 \\
\frac{\partial n_{Q}}{\partial t}=n_{Q-1} n_{e} \beta_{Q-1, Q}+n_{Q+1} n_{e}^{2} \alpha_{Q+1, Q}-n_{Q} n_{e} \beta_{Q, Q+1}-n_{Q} n_{e}^{2} \alpha_{Q, Q-1} \text { for } Q \geq 1
\end{gathered}
$$

where $\beta$ and $\alpha$ are the ionization and recombination coefficients [13], respectively,

$$
\begin{aligned}
\beta_{Q, Q+1}\left(E_{Q}, T\right) \sim & \frac{\Sigma_{Q+1}}{g_{Q}} \exp \left(-\frac{E_{Q}-\Delta E_{Q}}{k T}\right) /(k T)^{3}, \\
& \alpha_{Q, Q-1} \sim(k T)^{-9 / 2} .
\end{aligned}
$$

An analysis of these expressions shows that (i) the expansion time slowly increases (proportional to $n_{p l}^{-1 / 2}$ according to (9)) while the reaction times respond much faster as the density decreases. That means, as the plasma expands, it transitions from a state with many collisions and "reactions" near the emission site (equilibrium) to a state of much less collisions at greater distance from the emission site (nonequilibrium). The model approximation of charge state freezing makes use of this feature in that the ratios of charge states are calculated by the relatively simple system of Saha equations at the transition from equilibrium to non-equilibrium. Assuming sudden freezing, one can associate the charge state distribution measured far from the emission site (i.e. far from the cathode spot) with plasma conditions at the freezing zone. For point-like expansion in the absence of an external magnetic field this is roughly about $100 \mu \mathrm{m}$ from the spot center. This has been done for all metals of the periodic table of elements [14]. In doing so, one can use measured charge state distributions [15] to determine the electron temperature at the freezing zone, which is typically $3-4 \mathrm{eV}$. The measured charge state distributions 
could be approximately reproduced, however, for most elements, the measured distributions were broader than the calculated. Clearly, the model assumption of instantaneous freezing is rather crude. It is equivalent to assuming that the Damköhler number suddenly switches from $\mathscr{D} \boldsymbol{a} \gg 1$ to $\mathscr{D} \boldsymbol{a} \ll 1$ at a certain distance from the cathode spot center. Refinements are needed.

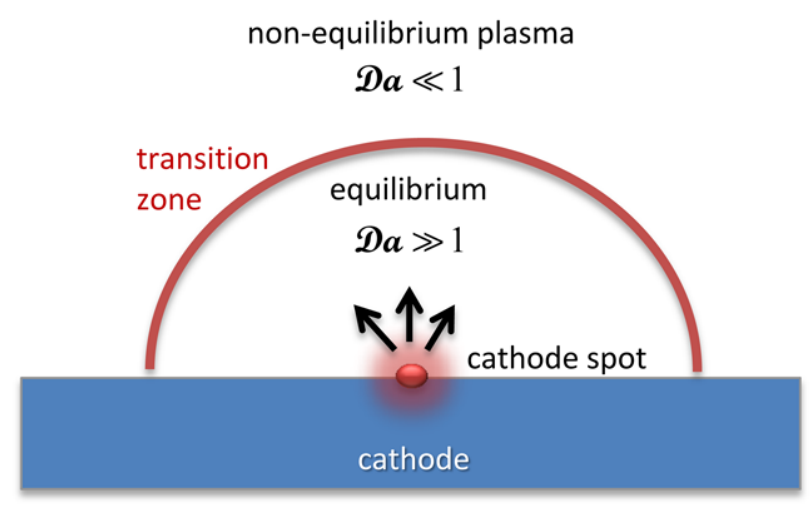

Figure 3. Schematic representation of plasma expansion from a cathode spot; the transition zone from ionization equilibrium to non-equilibrium is relatively well defined in the model of instantaneous freezing of the charge state distribution.

In a first refinement step, one could have a closer look at the freezing conditions for reactions between different charge states. So far it was assumed that all charge state ratios freeze at the same time (distance from spot). However, the ionization-recombination reactions are charge state dependent: ions of higher charge state leave the equilibrium system first [16]. This is not the only reason for the discrepancies between experimental observation and Saha-based calculations. The transition is certainly more gradual. In some cases the freezing model is not adequate, especially when the assumption of expansion from a point-like emission center is not applicable. External magnetic fields greatly modify plasma expansion and plasma flow, which has numerous implications. We will return to this point in section 8.

\section{Fractal cathode spots}

The different paths of the cathode material in the phase diagram are indicative for a more general pattern or property exhibited by the cathode processes, namely that the cathode spot is a fractal [17]. Fractals are mathematical or physical objects that are self-similar, which means they are invariant to multiplicative changes of scale [18]. A self-similar object appears (approximately) unchanged after increasing or decreasing the scale of measurement and observation. Self-similarity may be discrete or continuous, deterministic or probabilistic. To see the relation to cathodic arc phenomena, a few comments are made on fractals and self-similarity.

Power laws are an abundant source of self-similarity [19] because of their invariance to multiplication. Consider the homogenous power law

$$
f(x)=c x^{\alpha}
$$


where $c$ and $\alpha$ are constants. It is self-similar because rescaling-multiplication with a constantpreserves that $f(x)$ is proportional to $x^{\alpha}$, albeit with a different constant of proportionality. Therefore, a fruitful approach to identifying and modeling fractals is to look for power laws describing the object or physical phenomena. Power laws can be found in relation to cathode spot phenomena, most notable are power laws that describe the spectral power density of fluctuations ("noise") of (i) the arc burning voltage [20, 21], (ii) the ion current density at a substrate or probe, (iii) the light arriving at a suitable (fast) detector [22-24], (iv) the electromagnetic waves in the RF spectral region (we are all familiar with a crackling noise in radios when switching occurs - these are examples the cathode emission phenomena [25]). Other arc properties also show power laws, like the size distribution of macroparticles emitted from the cathode spot [17]. Last but not least, the patterns seen in light emission and left on cathode surface are "tree-branched" - a typical self-similar pattern $[24,26]$. Clearly, there is a relation to the apparent motion of cathode spots, which in the absence of a magnetic field can be modeled by random walk [27-29]. The fractal properties can be recorded using the streak camera technique (Fig. 4).
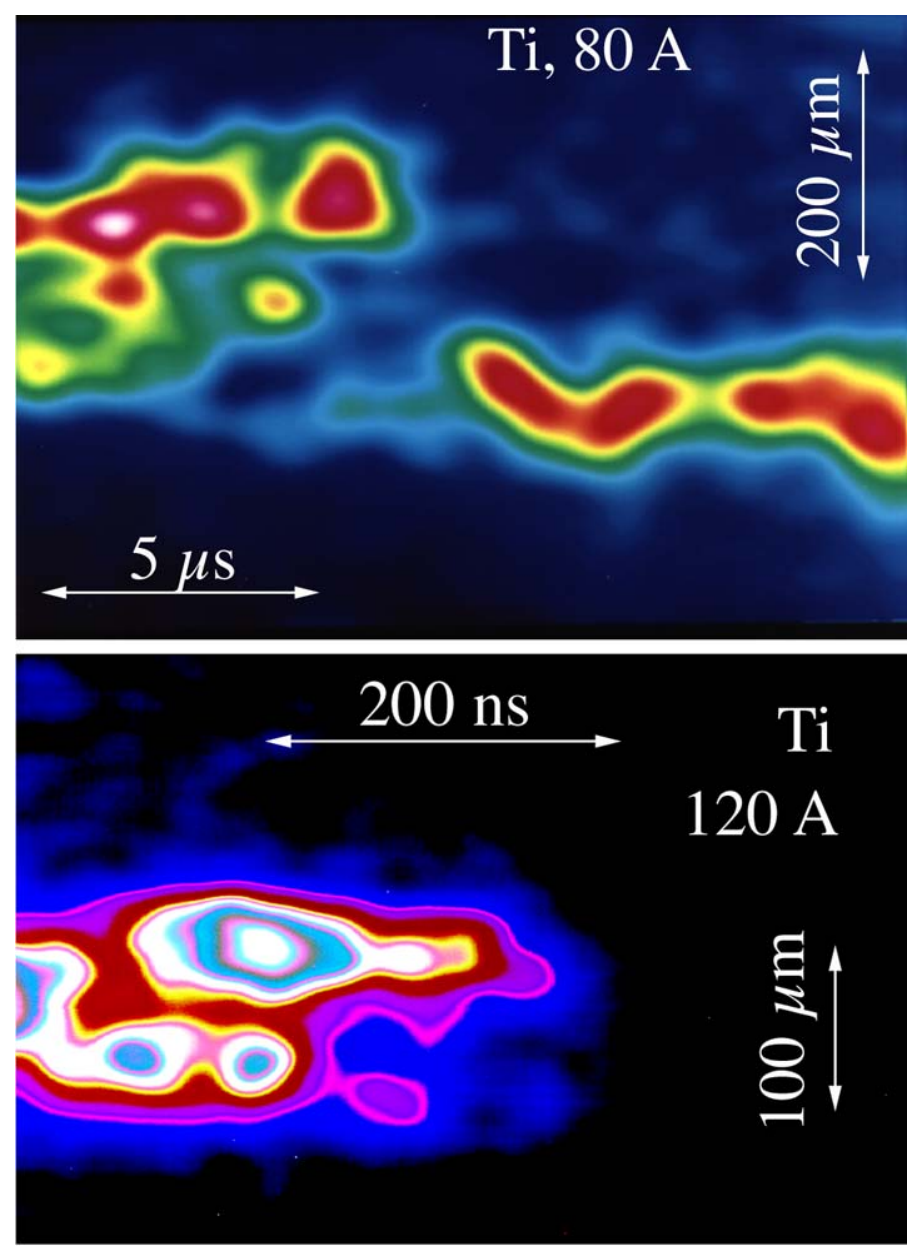

Figure 4. Two examples of false-color streak camera images showing spectrally integrated light intensity from emission sites on a titanium cathode in vacuum. The lower image features more than $10 \times$ greater time resolution compared to the upper yet the features look similar, which is evidence for self-similarity, a key fractal feature. More details on the technique can be found in [9, 30]. 
Random walk is a discrete fractal exhibiting stochastic self-similarity on large scales, but self-similarity is cut off as scales approach the elementary step width $s$ [17]. The well-known Brownian motion is the scaling limit of random walk. That means that if random walk occurs with very small steps, random walk becomes an approximation to Brownian motion, a fractal with the fractal (Hausdorff) dimension 2 [19]. In the presence of a magnetic field, the symmetry related to the spot ignition probability is broken and one observes an apparent spot motion into a preferred (steered) direction [6, 30, 31]. The fractal dimensional is then reduced, for example to 1.65 in the experiments of Kajita et al. [32].

The discussion is relevant to the starting point where the path of cathode material in the phase diagram was considered. Once we have realized that the cathode spot phenomena are fractal in nature, it becomes clear that questions like

- What is the current density of cathode spots?

- What is the burning voltage of cathodic arc?

- What is the lifetime of a cathode spot?

- What is the path of the cathode material in the phase diagram?

- What is the charge state distribution of the expanding plasma?

do not have a deterministic answers! Rather, a fractal approach is appropriate, where the actual time and space-resolved values show self-similarity depending on the resolution limits of the measuring instrumentation down to physical cutoffs.

While mathematical fractals show self-similarity at all scales, physical fractals have cutoffs. Considering spatial cutoffs, for example, one can readily see that that they are determined by the system size (large scale limit) and by the atomic and quantum nature (small scale limit).

The history of the cathodic arc research is rich in the search for cutoffs in the fractal model, even as this is not recognized or explicitly expressed in this way. For example, the search for spot lifetime was often seen as a search for the shortest possible process that leads to the formation of a cathode spot (as e.g. evident by a crater left on the surface) [9,33]. Another example is the search for the "true" power density which is generally a search to determine the "true" area of the emitting site since current and voltages are relatively easily measured [34,35]. Since the area changes as the spot evolves, this search was characterized by increasingly sophisticated diagnostic techniques with ever higher spatial and temporal resolution [23, 33, 36-38]. The power density is of great interest since any modeling of the spot phenomena needs to deal with the energetics of the processes, and theoreticians are looking to experimentalists for guidance what the "true" values are so theories could be tested on them [39].

A relevant concept in this context is the search for an elementary amount of the energy needed to form an explosive event. The explosive emission mechanisms has parallels to the theory of wire explosions [40], where the current density, $j$, and explosion delay time, $t_{d}$, satisfy

$$
\int_{0}^{t_{d}} j^{2} d t=\bar{h},
$$

where $\bar{h}$ is called the specific action whose value depends on the cathode material but is approximately independent of current density, wire cross section, or other discharge quantities. According to the ecton model [41-44], thermal runaway occurs on microprotrusions until they explode with a delay time associated with the material-specific action. The ecton is the "quantum" of an electron emission event or microexplosion. The explosions create microscopic defects including new microprotrusions, thereby 
facilitating sequences of many microexplosions which manifest themselves as the apparent cathode spot motion.

Although the light emitted by the plasma of an emission center exhibits typical fractal features in time and space, it can never be used to investigate possible substructures because the light from any physically relevant substructures, if they exist, are smeared-out due to the finite lifetime of excited states $t_{e x c}$ and the high velocity of the excited ions. For example, if the excited ion moves with the typical velocity of $10^{4} \mathrm{~m} / \mathrm{s}$, and the lifetime of the excitation is $10^{-9} \mathrm{~s}$, the spatial resolution is fundamentally limited to $v_{p l} t_{\text {exc }} \sim 10^{-5} \mathrm{~m}[23,45]$. Streak camera images show indeed no sharp features smaller than 10 $\mu \mathrm{m}$ on their spatial axes. Therefore, other techniques need to be employed that do not rely on light emission. Laser light absorption [46-48] and interferographic techniques [49, 50] indicated the existence of a cathode spot sub- or cell structure, which was long suspected [51] and discussed in the literature [23, $44,52,53]$. The individual microexplosion or "ecton" can be understood as the small-scale physical cutoff of a fractal vacuum arc model [17]. Yet, even the most advanced techniques have not yet yielded a clear cutoff: ever improved diagnostic techniques have revealed finer structured features and processes.

\section{Conventional and unipolar arcs}

The concept of initiation of emission sites, fractal model description, and many other features are applicable to "conventional" and "unipolar" vacuum arcs. Generally we deal with conventional arcs, where anode and cathode are connected to an arc power supply that provides the necessary voltage and current to sustain the arc discharge. The dense plasma is responsible for the fact that most of the burning voltage (as measured between anode and cathode) drops in a very thin sheath adjacent to the cathode surface. Since the plasma density is very non-uniform it is clear that the sheath thickness is also very non-uniform. In fact, it was argued that for the brief movement of a phase transition around the critical point " $C$ " (Fig. 1), the sheath does not exist at the location of the microexplosion [6]. This aside, and neglecting a number of other issues, the local sheath thickness can be roughly estimated by the Child law $[6,54]$

$$
S_{\text {Child }}=\frac{\sqrt{2}}{3} \lambda_{D}\left(\frac{2 e V_{c}}{k T_{e}}\right)^{3 / 4},
$$

where $V_{c}$ is the voltage drop of the cathode fall, and $\lambda_{D}$ is the Debye length (3). As the plasma density is high, for example close to a just-exploded emission site, the sheath is very thin and therefore the surface electric field

$$
E_{\text {surface }}=\beta_{\text {enhance }} E_{0}
$$

can be extremely high even as the voltage drop is only about $20 \mathrm{~V} ; E_{0}$ is the surface field if the cathode was perfectly flat and chemically clean and uniform, and $\beta_{\text {enhance }}$ is the field enhancement factor which is introduced $a d-h o c$ to describe the local enhancement due to geometrical and chemical peculiarities of the real surface such as asperities, grain boundaries, dust particles, or oxide layers. Field enhancement factors in the range 2-10 are quite common. However, higher values in the range 10-100 [55] and up to

$10^{3}$ [56] have been reported. In extreme cases of nanowires, $\beta_{\text {enhance }}$ can exceed $10^{4}$ [57]. A high electric 
surface field, of order $10^{8}-10^{9} \mathrm{~V} / \mathrm{m}$, will necessarily lead to thermal runaway of electron emission and plasma formation, as mentioned before. This perpetuates the ignition of emission sites [6, 58].

The situation can be surprisingly similar for emission sites on an electrical isolated (floating) but conducting wall or shield, leading to "unipolar" arcs [59]. A floating object assumes a negative floating potential relative to the plasma potential in order to balance the electrical current of the more mobile negative plasma electrons against the less mobile positive ions [59, 60]

$$
V_{f} \approx \frac{k T_{e}}{2 e} \ln \left(\frac{m_{i}}{2 \pi m_{e}}\right) .
$$

Provided that the sheath is very thin and the floating potential is of order the arc burning voltage or greater, an electron emission site may ignite very much the same way it does for "conventional" vacuum arcs [61]. The emission site provides dense plasma of low impedance, which can short-out the sheath voltage and the "unipolar" arc will stop. However, the discharge may persist if the bulk plasma is heated by external means, like in a Tokamak. The floating wall can be simultaneously an electron emitter at the emission site (cathode function) and a receiver of hot plasma electrons (anode function) [62]. Unipolar arcs can erode the floating wall and produce a flux of highly undesirable dense plasma of the wall material, contaminating the fusion plasma. Therefore, unipolar arcs are subject of renewed interest [63-65].

\section{Cohesive Energy Rule}

Early in the research of vacuum arcs it became clear that similar cathode materials behave similarly. For example, refractory metals tended to make plasmas with the highest charge states, whereas large craters and large macroparticles were observed when using cathode material of low melting or boiling point. Brown and co-workers established the empirical relation [15, 66]

$$
\bar{Q}=0.6+3.8 \times 10^{-4} T_{b o i l}[K]
$$

between the mean ion charge state number, $\bar{Q}$ (measured about $150 \mu$ s after arc start), and the boiling temperature (in Kelvin) of the cathode material. Later we will refer to this as "Brown's Rule." While such empirical relation is helpful, the underlying physics remained obscure. In the last 20 years, much research focused on how the ion charge state distributions develop and what the reason for the strong material dependence is.

One step toward physical modeling of the processes is to formulate empirical rules based on energy considerations as opposed to rather arbitrary physical parameters such as boiling temperature or thermal conductivity. After all, we have energy conservation, but no conservation laws for temperature or thermal conductivity. In our strongly time dependent system we should include the energy input per time, or power, which essentially means to consider the time-dependent, self-establishing arc burning voltage. Accordingly, the Cohesive Energy Rule for vacuum arcs can be considered as an empirical relationship between the cohesive energy of the cathode material and the arc burning voltage because the local power density, $p$, is directly related to the arc burning voltage, via the local current density, $j$, by

$$
p=j V .
$$

The Cohesive Energy Rule states that the burning voltage depends approximately linearly on the cohesive energy. The cohesive energy is defined at as energy needed to separate an atom from its 
neighbors in a solid and to move it to infinite distance; for values see Table I in [11], for example. Based on experiments at Berkeley’s vacuum arc ion source, the Cohesive Energy Rule was quantified as

$$
V=V_{0}+A E_{C E}
$$

with $V_{0} \approx 14.3 \mathrm{~V}$ and $A=1.69 \mathrm{~V} /(\mathrm{eV} / \mathrm{atom})$ [20, 67] (Fig. 5).

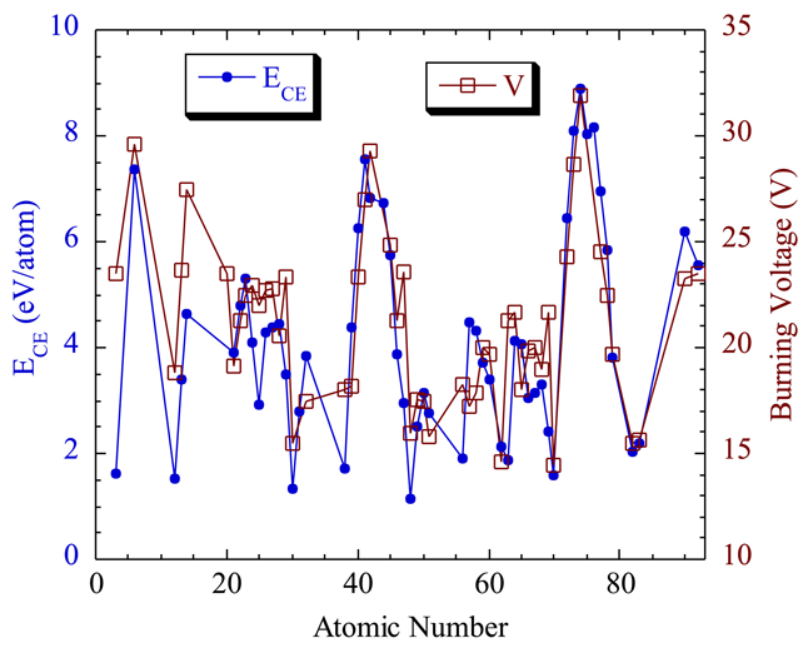

Figure 5. Cohesive energy and vacuum arc burning voltage for most conducting elements of the Periodic Table (adapted from [67]).

In order to accomplish the phase transition from the solid to the plasma, energy must be invested, and the cohesive energy represents, roughly speaking, the energy needed to reach the expanded vapor phase. A fraction of the invested power is used to facilitate phase transitions. Another fraction is used to produce the various ion charge states and to accelerate the plasma away from the cathode. Materials with high cohesive energy tend to produce plasmas with higher electron temperature and related higher charge state. These less-than-rigorous arguments are supported by surprisingly good correlations as indicated in Figs. 5 and 6.

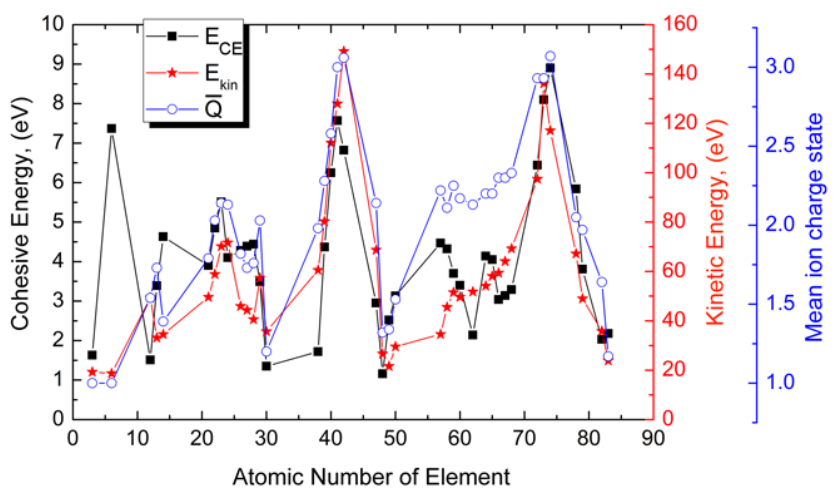

Figure 6. The mean charge state and the most likely kinetic energy of ions show a correlation to the cohesive energy and a periodicity according to the Periodic Table of Elements (adapted from [11]). 
Another reason to justify the Cohesive Energy Rule is that many physical properties of the cathode material show periodicity as expressed in the periodic table of the elements. Therefore, the periodicity of the cohesive energy is a proxy for the periodicity of other quantities such as melting and boiling temperatures or ionization energies [67]. In this sense, Brown's Rule for the mean ion charge state number, equation (20), is directly related to the Cohesive Energy Rule.

\section{Use of high purity metal, oxidized, semiconducting, or alloy cathodes}

It has been long recognized that the surface conditions of the cathode are very important yet difficult to control or reproduce. For example, Cobine in 1938 stresses the role of oxides and metal impurities for the reignition of arc spots [68]. "Type 1" and "type 2" cathode spots were introduced by Djakov and Holmes [69] and later associated with surface conditions by Jüttner and coworkers [70, 71]. At about the same time, Proskurovsky found that "the formation of new emission centers below the plasma of the cathode spot by a mechanism involving explosion of microscopic fine tips can occur at distances similar to $10^{-4} \mathrm{~cm}$ from the primary emission center, while a mechanism involving the breakdown of nonmetallic inclusions and films on the cathode can explain the formation of new emission centers at distances out to $10^{-2} \mathrm{~cm}$ " [72]. The role of surface conditions is emphasized in most reviews on cathode spots [6, 29, 43, 53, 73]. Consistently, the plasma generated by spots on oxidized or nonconditioned cathode surfaces contains large amounts of non-metal ion species. Using pulsed arcs of various repetition rates, it has been demonstrated that the fraction of gas ions in the plasma is clearly related to the time it takes to form an adsorbate layer from residual gas [74].

Semi-metals and semiconductors represent their own class of cathode materials. In contrast to metals, high current causes a very significant voltage drop inside in the cathode (compared to the voltage drop between anode and cathode). This is associated with significant Ohmic heating, which in cases of semiconductors actually enhances conductivity and thereby reduces the need for new spot formation. The velocity of the apparent spot motion is therefore smaller than with metals. Spots on those cathodes may be assigned their own spot type (type 3) [6].

Another interesting situation arises when the cathode is made of more than one metal. In light of the Cohesive Energy Rule ( $c f$. section 6) it turns out that the charge states of the constituent with low cohesive energy are enhanced, compared to the pure metal situation, and the ion charge states of the material of higher cohesive energy are relatively reduced [75-78]. One may also look at this in terms of the electron temperature that can be associated with ionization: it is about the average value of the temperatures one would expect in the pure metal cases [14]. Of special interest to ion sources, the effect can be utilized to enhance the ion charge states by alloying the desired material with another material of higher cohesive energy.

\section{Effects of the magnetic field on plasma expansion and ion charge states}

External magnetic fields are applied in many practical applications of cathodic vacuum arcs (or cathodic arcs in general). This is to control process parameters like plasma density, to steer the apparent spot motion, to guide plasma in a macroparticle filter and to control plasma impact on a substrate, and, last but not least, to enhance the ion charge states.

The shift to higher charge states by a magnetic field has been extensively documented in the literature [79-87]. The strength of the magnetic field is in most cases not sufficient to "magnetize" ions: 
only the electrons are magnetized. By "magnetization" we mean that (i) the gyration radius of the charged particle of charge $Q e$ and mass $m$

$$
r_{L}=\frac{v_{\perp} m}{|Q| e B}
$$

is small compared to a characteristic length of the system (such as the inner radius of a macroparticle filter, or the cathode-anode distance), and (ii) the charged particle can execute many gyrations around the magnetic field line before experiencing a collision. In (23), $v_{\perp}$ is the velocity component perpendicular to the magnetic field (induction) vector $\mathbf{B}$. Magnetized particles tend to move along magnetic field lines (motion of the gyration center, i.e. the motion is averaged over the gyration), which is utilized in macroparticle filters among other apparatus. Cross-field motion is facilitated via collisions and collective modes (micro-instabilities).

Near the emission center we implied that many collisions occur, which can be associated to a kinetic pressure

$$
p_{\text {kin }}=\gamma \frac{I_{\text {arc }}}{r^{2}} k T
$$

where we again used the relation (10). The local magnetic pressure can be determined from

$$
p_{\text {mag }}=\frac{B^{2}}{2 \mu_{0}}
$$

where $\mu_{0}$ is the permeability of vacuum. The second condition of magnetization implies $p_{\text {mag }}>p_{\text {kin }}$, which means than plasma electrons are only magnetized for distances

$$
r>\frac{1}{B}\left(2 \mu_{0} \gamma I_{a r c} k T\right)^{1 / 2}
$$

To illustrate this, let's consider a typical temperature of $3 \mathrm{eV}$, an arc current of $100 \mathrm{~A}$, and a magnetic induction of $100 \mathrm{mT}$. We obtain that the plasma electrons are magnetized and follow magnetic field lines if their distance from the emission center is greater than about $5 \mathrm{~mm}$. The electron gyration radius is of order $10 \mu \mathrm{m}$. Since the plasma is not expanding freely, the rate of expansion is less, the characteristic time of expansion is much longer and the Damköhler number does not drop as rapidly. Freezing of charge state ratios is not occurring, or at least not at the rate of free expansion. Additionally, the most energetic electrons, capable of causing ionizing collisions, are effectively confined such that they have a much higher probability of actually causing an ionizing collision. These effects lead to enhanced ionization compared to the free expansion case. The reduced expansion and flow of plasma along magnetic field lines can be readily seen in macroparticle filters of open architecture [88, 89] (Fig. 7). 


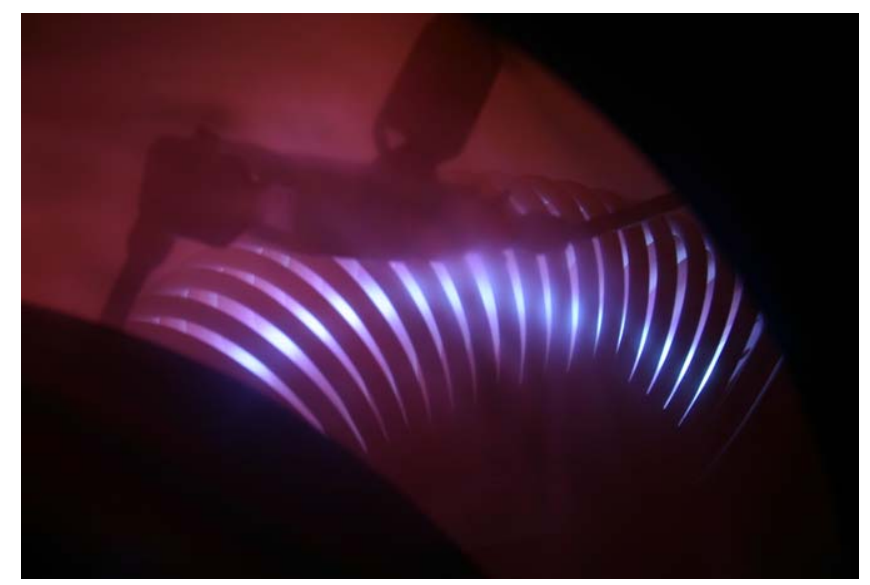

Figure 7. Illustration of plasma confinement and guiding in an open-architecture macroparticle filter; the arc source with a $6.25 \mathrm{~mm}$ diameter cathode rod is on the right side, and the plasma is streaming to the left toward a substrate. The visible plasma stream has a diameter of about $10 \mathrm{~mm}$; for more information see [88, 89].

Yet another effect is that electrons are magnetically shielded from reaching the anode, unless the magnetic field lines directly intersect emission site and anode surface. An additional voltage drop between anode and cathode develops setting up an electric field that assists electron transport to the magnetically shielded anode. Even as the additional voltage is generally less than $40 \mathrm{~V}$, the value should be seen in comparison to the arc burning voltage of magnetically unimpeded arcs, which are in the range from 15 to $29 \mathrm{~V}$ depending on the material [11], see equation (22). Since the power density (21) is proportional to the voltage, the enhancement of the arc voltage upon application of an external magnetic field provides the mechanism by which additional energy is provided for producing higher ion charge states. A significant enhancement of the arc burning voltage is consistent with a significant shift to higher ion charge states [11, 79-87].

\section{Charge exchange collisions}

Early papers of the 1960s and 70s reported about the existence of multiple charge states in vacuum arc plasmas, which are characteristic for the cathode material [90-93]. More detailed investigations revealed that pulsed arcs have higher charge states compared to dc arcs [94-96], or, as Galvin and co-workers put it, "There is an interesting early-time transient behavior when the arc is first initiated and the arc current is still rising, during which time the ion charge states produced are observed to be significantly higher than during the steady current region that follows” [97]. The characteristic time for this "early-time transient behavior" is of order of several $10 \mu \mathrm{s}$ and its origin was subject to many investigations of cathodic arcs in vacuum [94-102] and with background gas [103, 104].

Two arguments have crystallized to be responsible for higher charge states seen early after discharge ignition: One is associated with the observation that, at the very beginning of a discharge, the voltage between anode and cathode is high and the discharge is still in its spark phase (see section 10). The other argument, and much more relevant for the time scale of change observed, is the evolution of a neutral gas or vapor background as the discharge operates. This requires more elaboration.

Already in 1975, Daalder studied several cathode materials and states that "ions and neutrals differ in origin of generation, the cathode spots being the centres of fully ionized metal vapour, while neutrals are formed in surrounding areas" [105]. The relevance of this finding for the evolution of ion 
charges states was overlooked for over two decades. The charge exchange reaction between a metal ion of charge state $Q$ and a neutral atom $A$ conserves charge but leads to a reduction of the average ion charge state (since neutrals, $Q=0$, are not included in the definition of the mean ion charge state) [99]:

$$
M e^{Q+}+A \Rightarrow M e^{(Q-1)+}+A^{+}, \quad Q=1,2,3, \ldots
$$

The cross section for charge exchange collisions is large compared to the cross sections of most other types of collision, and therefore charge exchange collisions are very important, even at moderate densities of neutrals. The sources of neutrals are manifold and include evaporating macroparticles, vapor from still-hot, previously active emission sites, desorption of gas from surfaces exposed to plasma and its radiation, and atoms returning to the plasma due to less-than-complete condensation of the metal plasma upon arrival on a surface. The latter effect alone is important in the sense that even a fully ionized plasma flow is a source of neutrals!

Evidence for the last point can be found in "optical flares" as reported by Tarrant and coworkers [106] and more recently also studied in our laboratory [107]. Such "flares" manifest themselves as clear intensity enhancement of neutral emission lines close to the surface exposed to flowing cathodic vacuum arc plasma (Fig. 8).

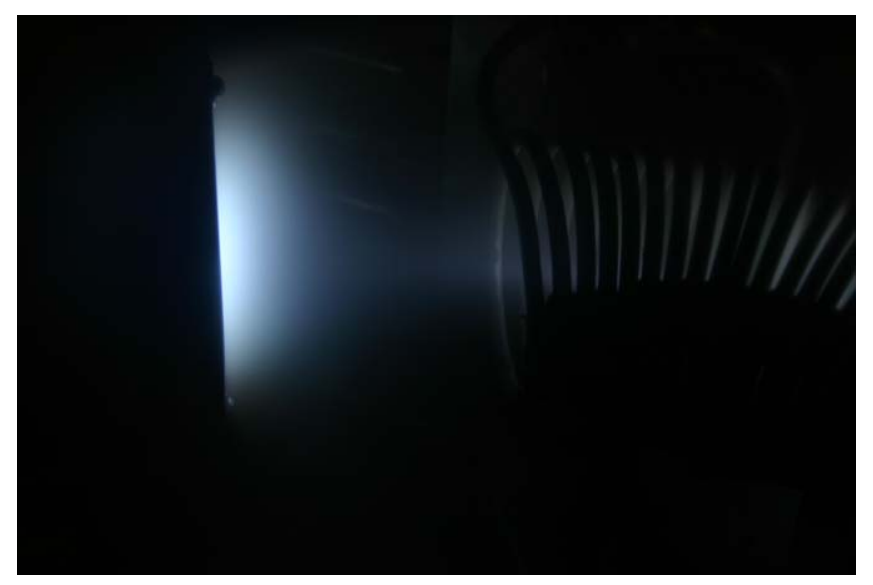

Figure 8. Open-shutter photograph through the vacuum window showing the enhanced optical emission when a surface (left) is hit by cathodic vacuum arc plasma (steaming from the right); for details see [107].

The "flares" are evidence of the less-than-unity sticking coefficient, $S_{c}$, of vacuum arc ions [108]. Even as only a small percentage of ions do not stick, the flux of atoms (i.e., neutralized former ions) from the surface causes a very relevant density according to

$$
n_{a}=\left(1-S_{c}\right) n_{i} v_{i} / v_{a}
$$

since the characteristic atom velocity $v_{a}$ is much smaller than the characteristic ion velocity $v_{i}$. Nonsticking necessarily produces neutrals, and charge exchange reactions (27) occur, predominantly reducing the fraction of higher charged ions and producing more singly charged ions, in agreement with measurements $[100,102]$.

Several other examples should be mentioned here that clearly support this interpretation. In the first, sophisticated optical and spectroscopic measurements were used on a liquid-metal cathode of a 
gallium-indium alloy under ultrahigh-vacuum conditions. Broadened lines of gallium and indium single-, double- and higher charged ions dominated the spectrum at the beginning of the pulsed discharge, and atomic lines appeared with a delay of several hundreds of nanoseconds. The intensity of all lines then decayed but those of atoms at a much slower rate [109].

The next example concerns the peculiarly different evolution of charges states for the lowmelting point materials bismuth and lead. Most of their physical properties are very similar, including melting point $\left(T_{\text {melt }}(P b)=600.6 \mathrm{~K}, T_{\text {melt }}(B i)=544.7 \mathrm{~K}\right)$, boiling point $\left(T_{\text {boil }}(P b)=2013 \mathrm{~K}\right.$ and $\left.T_{\text {boil }}(B i)=1833 \mathrm{~K}\right)$, and cohesive energy $\left(E_{C E}(P b)=2.03 \mathrm{eV}, E_{C E}(B i)=2.18 \mathrm{eV}\right)$. According to Brown's Rule (20) one would expect that the mean ion charge state of bismuth is slightly lower than lead's, whereas the Cohesive Energy Rule suggests the opposite. None of those rules refer to the evolution of the charge state distribution. Time resolved measurements (Fig. 9) showed that the mean charge state extrapolated to times shorter than $50 \mu$ s after arc ignition is slightly higher for $\mathrm{Bi}$, in agreement with the Cohesive Energy Rule [101]. For later times, the mean charge states drop for both $\mathrm{Pb}$ and $\mathrm{Bi}$, but much more significantly for $\mathrm{Bi}$. Brown's Rule [15, 66] was established based on experimental data taken about $150 \mu$ s after arc ignition, and indeed, at that time, bismuth has slightly lower mean charge state than lead (Fig. 9).
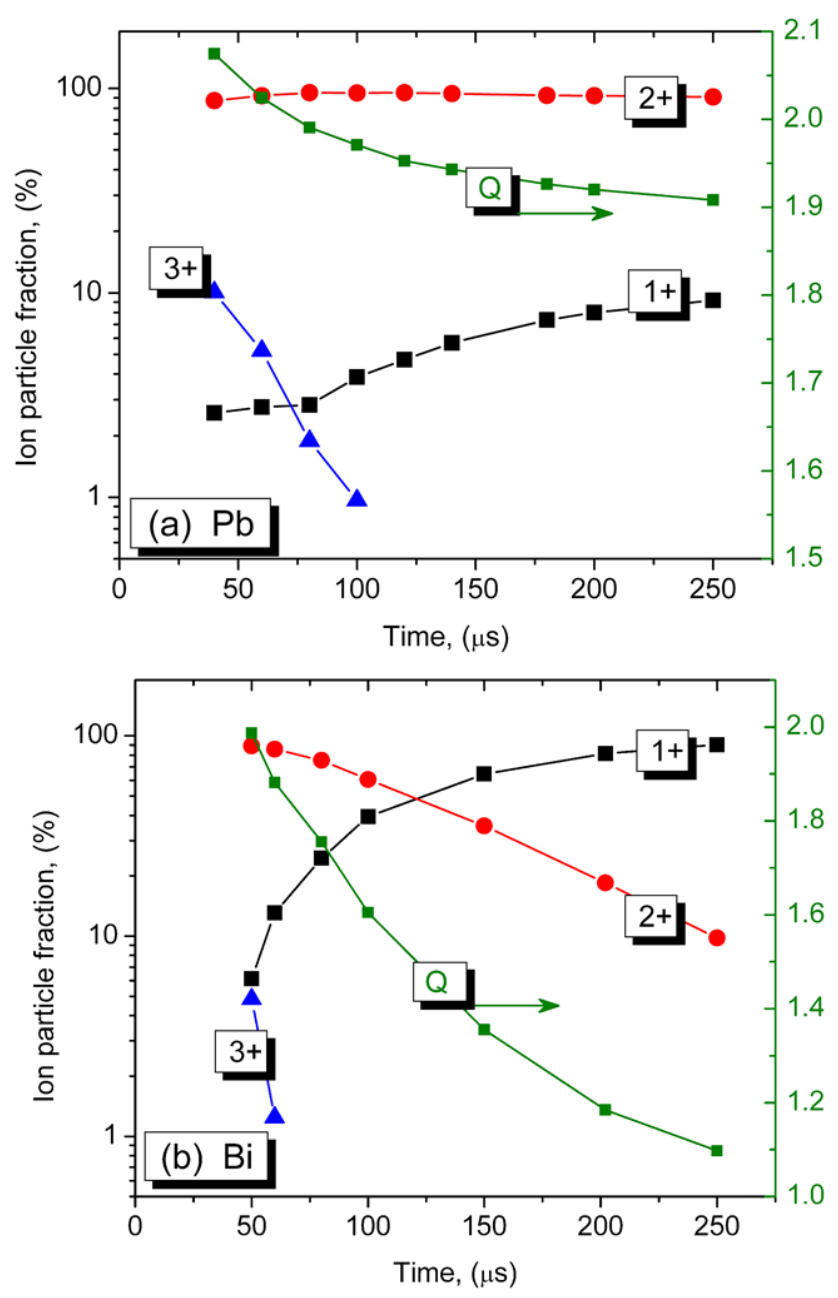

Figure 9. Evolution of the ion charge state distribution of cathodic vacuum arcs for (a) lead plasma and (b) bismuth plasma. Arc current of $300 \mathrm{~A}$, ion extraction (analysis) about $10 \mathrm{~cm}$ from the cathode; from [101]. 
The reason for the much stronger decay of the mean ion charge state for bismuth can be found in charge exchange collision with metal neutrals. Bismuth has a much smaller thermal conductivity and much higher vapor pressure than lead, which leads to the evolution of a much higher background vapor [101].

If charge exchange collisions with neutrals are really the reasons for the reduction of charge states, more of such collisions should occur as the ions travel from the cathode spot region, and a corresponding charge state reduction should be seen. This was tested in a special setup of the time-offlight instrument equipped with a movable cathode: the mean ion charge state indeed decreased as indicated in Fig. 10.

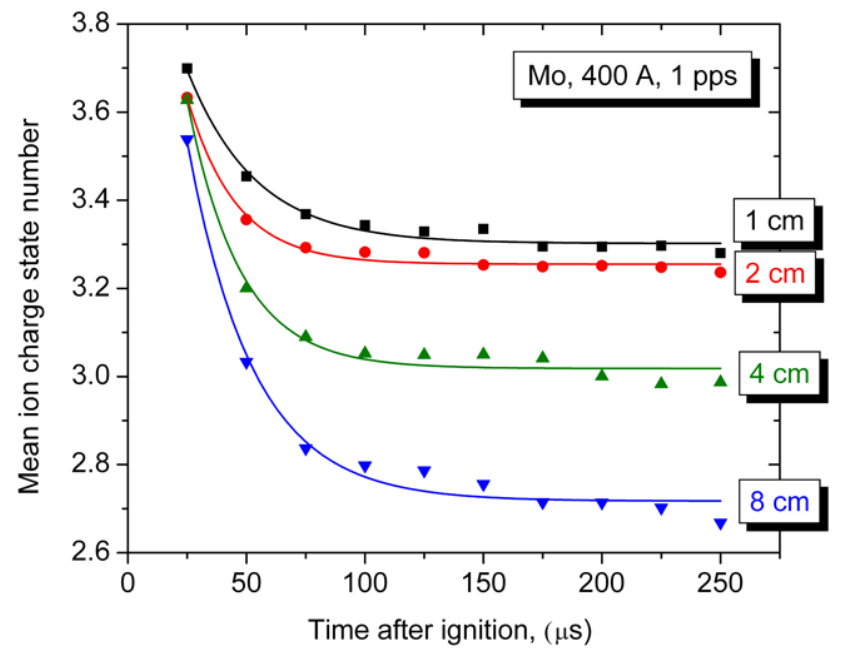

Figure 10. Mean ion charge state number for molybdenum vacuum arc plasma as a function of time after arc ignition for different distances between cathode surface and the ion extraction grid, which is effectively the location of ion analysis; from [102].

Charge exchange does not only occur with neutrals of the same element (i.e. the metal the cathode is made of) but with gas atoms, should they be present. Most vacuum systems are not ultra-high vacuum (UHV) systems and therefore water is adsorbed on walls and present as vapor when desorbed. Additionally, one may intentionally add gases, for example when the goal is to produce compound films using the cathodic arc process [6, 110-113]. Besides electron impact ionization, charge exchange collisions produce gas ions. Gas ions, as opposed to metal ions, can represent a very large fraction of the plasma or even become the dominant ion species [114-118].

Extrapolating time-dependent charges state distributions to $t \rightarrow 0$, i.e. a time when the spot plasma expands into space not yet filled with neutrals, the charge states are significantly higher than reported in survey tables [11, 14, 15, 66, 90, 91]. This suggests that the ion charges states produced during the explosive process in cathode spots are much higher than observed far from the spot. One may suspect that especially for dc arcs, when a background of neutrals is fully developed, the observed and used ion charge states are actually significantly lower than what is produced at the non-stationary cathode spots. 


\section{Vacuum arc and vacuum sparks}

Short-pulse cathodic vacuum arcs are sometime loosely labeled as "vacuum sparks" which, strictly speaking, is not correct. Vacuum arcs are characterized by their typical low anode-cathode voltage, Eq. (22), which can be enhanced by magnetic fields, for example, as discussed in section 8 . Yet, even enhanced arc voltages are generally less than $100 \mathrm{~V}$. Vacuum sparks, in contrast, are characterized by a high voltage, which can be 10 s of $\mathrm{kV}$, for example. Such high voltage between electrodes cannot be sustained and therefore vacuum sparks are always transient phenomena.

Often, a short spark phase can be observed when a cathodic vacuum arc is initiated. For example, a $\mathrm{kV}$-pulse generator with a few ns rise time was applied to a very short $(100 \mu \mathrm{m})$ anode-cathode gap to produce an 800 ns pulsed arc; the voltage between anode and cathode remained high for about $20 \mathrm{~ns}$, which is the time the plasma needed to bridge the electrode gap [46, 47].

Driven by low impedance pulse power generators, vacuum sparks show very high current (often 10s of kA) up to the point where pinching occurs caused by the high magnetic self-field. A phenomenon called radiative collapse leads to the formation of high density plasma hot spots that emit $\mathrm{x}$-rays characteristic of highly charged ions, e.g. helium-like Fe XXIV [119], molybdenum Mo XL [120], or magnesium Mg X [121]. Such phenomena are very different from vacuum arcs.

\section{Additional ionization of cathodic vacuum arc plasma}

In an attempt to make higher charges states available for particle accelerators, ion implantation and surface modification, short pulse arcs with a brief initial spark phase can be utilized and further optimized by using pulsed high current, additional external magnetic fields, and good vacuum practices (less gas adsorbates). Early experiments were done in the 1950s [122]. In more recent studies, pulsed ion beams were produced from plasmas in the spark-arc transition region containing multiply charged ions up to $11+[123,124]$ (Fig. 11).

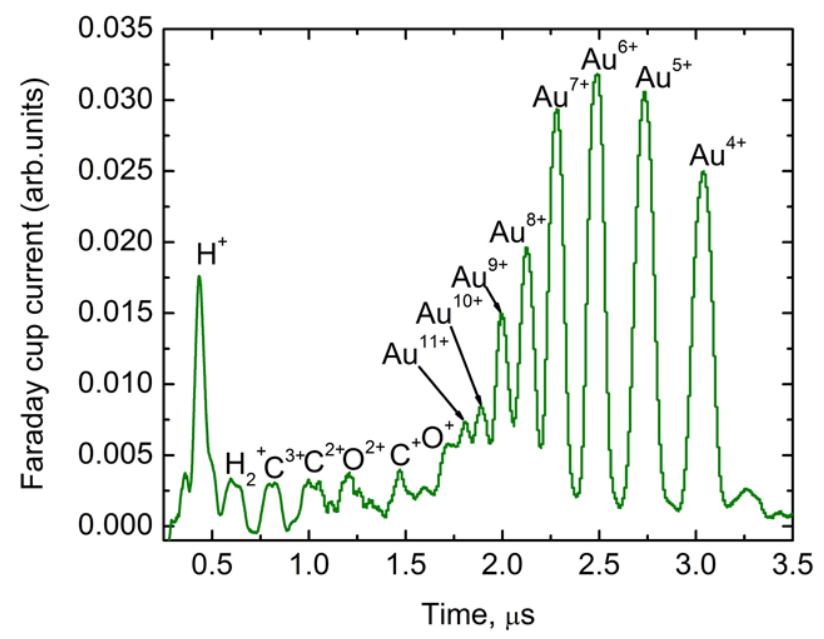

Figure 11. Typical time-of-flight spectrum of a pulsed vacuum discharge in the spark-arc transition region with a gold cathode; peak current was $1.5 \mathrm{kA}$, discharging a low-inductance $450 \mathrm{nF}$ storage capacitor charged to $4 \mathrm{kV}$; from [124]. While the very first, small peak before the $\mathrm{H}^{+}$is electromagnetic noise, the multiple charge states of gold are clearly discernible. 
It appears very difficult to extract higher charge states for fundamental reasons related to recombination [124]. Systematic studies for many elements showed that the mean charge state number is typically shifted to higher values by only about 1-2 [125].

Further charge states enhancements for ion beam formation have been demonstrated for dedicated ion sources using magnetic compression and electron-beam ionization of the vacuum arc plasma in zMevva [126-128] and e-Mevva configurations [127, 129-131]). Alternatively, microwave-based electron cyclotron resonance (ECR) ionization can be used to shift the average charge state, e.g. from typically $2+$ to up to 7+ for platinum [132-134].

A simple approach to temporally enhance ion charges states is to superimpose current pulses on the arc current by increasing the applied voltage [135]. Each current pulse is driven by a temporary spike in the arc burning voltage and power. While this technique leads to only relatively small changes of charges states (similar to the study [125]), it was effectively used by Yushkov and coworkers to determine ion drift velocities in vacuum arc plasmas [136]. However, in their original work [136], a systematic error due to ion optics effects distorted the results especially for light ions. The authors then developed an improved technique a couple of years later to obtain generally accepted values [11]. Interestingly, the ion velocity distribution function for each material exhibited only one peak, indicative that all ion charge states of a material move with approximately the same velocity or kinetic energy [137]. This is an indication for the hydrodynamic nature of ion acceleration, as opposed to an electrostatic acceleration that would lead to kinetic energies proportional to the charge states [11].

Practically all the vacuum arc or "Mevva" type ion sources are broad beam sources that do not have mass/charge selection. The time-of-flight and similar diagnostics generally indicate two groups of ions, namely the multiply charged metal ions and lower-mass lower-charge gas ions. The latter usually stem from adsorbates on surfaces of the discharge system. Occasionally very high metal charges states such as $\mathrm{Au}^{19+}$ have been claimed to be extracted [128, 138, 139], however, it is much more likely that those ions are actually low ion charge states of desorbed gases. This statement is based on both theoretical considerations of ionization and recombination [124] as well as my own practical attempts using pulsed vacuum discharges up to $75 \mathrm{kA}$, which led to extracted charge states of "only" up to 7+ [140, 141].

\section{Summary and Conclusions}

The ion charge state distributions in vacuum arc plasmas are material dependent but not fixed as some older literature may suggest. Rather, the ratios of ion charge states undergo an evolution as the plasma is formed and expands from the cathode spot region, where it is then used for a variety of applications such as in ion accelerators, in vacuum arc switches, for surface modification and deposition of coatings. Decades of research can be summarized in the following main points:

1. Plasma generation occurs at cathode spots of extreme but variable power and current density best described by a fractal model. The ecton model of repetitive microexplosions is compatible with a fractal approach in that ectons (the smallest unit of microexplosions) represent the small-scale physical cutoff of self-similarity.

2. Non-ideal plasma effects such as pressure ionization play a role as the cathode material transitions from a solid to dense plasma, circumnavigating the critical point in the density-temperature phase diagram. The path of the material in the phase diagram is not fixed but rather subject to change depending on the development stage of the emission site. Such change is consistent with a fractal approach. 
3. The cohesive energy rule provides good guidance on estimating charge state distributions. The most complete compilation of charge state distributions (as observed about 150-200 $\mu$ s after arc ignition in 10-20 cm distance from the cathode) was published in [11]. It is reproduced with some minor additions in Appendix B of [6]. In the case of alloy cathodes, the plasma has one common effective electron temperature leading to a relative enhancement of the ionization of the material with low cohesive energy and a relative reduction of ionization of the material of high cohesive energy.

4. Charge exchange collisions of multiply charged ions with neutrals reduce the average ion charge states. As the neutral density itself is a function of the plasma development, such reductions are relatively slow (10s or 100s of microseconds after arc ignition) compared to the changes of the emission sites themselves (usually less than $1 \mu \mathrm{s}$ ). Extrapolation of charge state distributions to conditions without neutrals, or back close to the emission site, suggests that the ion charge states at the emission site are significantly higher than those actually utilized in applications.

5. Strategies for obtaining higher charge states for applications include reducing the neutral density, using magnetic fields, or apply additional ionization schemes such as electron-beam or electron cyclotron resonance ionization known from other types of ion sources.

6. Vacuum arcs should not be confused with vacuum sparks. Not the pulse duration but the voltage between anode and cathode should be used to distinguish them. Vacuum sparks are transient, high voltage, high current discharges that in extreme cases exhibit pinching, radiative collapse, and x-ray emission from highly ionized ions (e.g. helium-like $\mathrm{Mo}^{40+}$ ). Such ions are very short-lived and therefore cannot be extracted. Experiments using pulsed arcs (including a short spark phase) with magnetic field resulted in higher charge states up to about 10+. Some reports of much higher extracted ion charges (like $18+$ ) appear to be based on a misinterpretation of lighter and lower charged gas ions.

The initial question, triggered by the original publication [7], was whether or not the lowering of the ionization energy in dense plasmas (pressure ionization) is responsible for the high fraction of multiply charged ions in vacuum arc plasmas. The conclusion of the original paper was: yes, at least to some degree. The years of research suggest that, while the effect is likely to be present, other effects appear to be much more important in the establishment of observable plasma parameters.

\section{Acknowledgments}

Many of the here-collected results are based on ideas and contributions by close friends and colleagues. Running the risk of offending those not mentioned, I gratefully acknowledge the input over the years from just a few: B. Jüttner, E. Hantzsche, P. Siemroth, T. Schülke, I. Brown, E. Oks and G. Yushkov. This work was supported by the U.S. Department of Energy under Contract No. DE-AC02$05 \mathrm{CH} 11231$. 


\section{References}

[1] E. Hantzsche, "Mysteries of the arc cathode spot: A retrospective glance," IEEE Trans. Plasma Sci., vol. 31, pp. 799-808, 2003.

[2] S. Dushman, "Thermionic emission," Rev. Mod. Phys., vol. 2, pp. 381-476, 1930.

[3] R. H. Fowler and L. Nordheim, "Electron emission in intense electric fields," Proc. Roy. Soc. (London), vol. A119, pp. 173-181, 1928.

[4] E. L. Murphy and R. H. Good Jr., "Thermionic emission, field emission, and the transition region," Phys. Rev., vol. 102, p. 1446, 1956.

[5] E. A. Litvinov, G. A. Mesyats, and D. I. Proskurovskii, "Field emission and explosive emission processes in vacuum discharges," Sov. Phys. Usp., vol. 26, p. 138, 1983.

[6] A. Anders, Cathodic Arcs: From Fractal Spots to Energetic Condensation. New York: Springer, 2008.

[7] A. Anders, S. Anders, A. Förster, and I. G. Brown, "Pressure ionization: its role in metal vapor vacuum arc plasmas and ion sources," Plasma Sources Sci. Technol., vol. 1, pp. 263-270, 1992.

[8] B. E. Djakov and R. Holmes, "Cathode spot division in vacuum arcs with solid metal cathodes," $J$. Phys. D: Appl. Phys., vol. 4, pp. 504-509, 1971.

[9] B. Jüttner, "Nanosecond displacement times of arc cathode spots in vacuum," IEEE Trans. Plasma Sci., vol. 27, pp. 836-844, Aug 1999.

[10] S. Anders and A. Anders, "Frozen state of ionization in a cathodic plasma jet of a vacuum arc," $J$. Phys. D: Appl. Phys., vol. 21, pp. 213-215, 1988.

[11] A. Anders and G. Y. Yushkov, "Ion flux from vacuum arc cathode spots in the absence and presence of magnetic fields," J. Appl. Phys., vol. 91, pp. 4824-4832, 2002.

[12] V. A. Ivanov, B. Jüttner, and H. Pursch, "Time-resolved measurements of the parameters of arc cathode plasmas in vacuum," IEEE Trans. Plasma Sci., vol. 13, pp. 334-336, 1985.

[13] L. M. Biberman, V. S. Vorobev, and I. T. Yakubov, Kinetics of the Nonequilibrium LowTemperature Plasma. Berlin: Springer, 1987.

[14] A. Anders, "Ion charge state distributions of vacuum arc plasmas: The origin of species," Phys. Rev. E, vol. 55, pp. 969-981, 1997.

[15] I. G. Brown, "Vacuum arc ion sources," Rev. Sci. Instrum., vol. 65, pp. 3061-3081, 1994.

[16] A. Anders, "Plasma fluctuations, local partial Saha equilibrium, and the broadening of vacuum-arc ion charge state distributions," IEEE Trans. Plasma Sci., vol. 27, pp. 1060-1067, 1999.

[17] A. Anders, "The fractal nature of cathode spots," IEEE Trans. Plasma Sci., vol. 33, pp. 1456-1464, 2005.

[18] B. B. Mandelbrot, The Fractal Geometry of Nature. New York: W.H. Freeman and Company, 1983.

[19] M. Schroeder, Fractals, Chaos, Power Laws: Minutes from an Infinite Paradise, 8th ed. New York: W.H.Freeman and Company, 2000.

[20] A. Anders, E. M. Oks, and G. Yu.Yushkov, "Cathodic arcs: Fractal voltage and cohesive energy rule," Appl. Phys. Lett., vol. 86, pp. 211503-1-3, 2005.

[21] L. Ryves, D. R. McKenzie, and M. M. M. Bilek, "Cathode-spot dynamics in a high-current pulsed arc: A noise study," IEEE Trans. Plasma Sci., vol. 37, pp. 365-368, 2009.

[22] A. Anders, S. Anders, B. Jüttner, H. Pursch, W. Bötticher, and H. Lück, "Vacuum arc cathode spot parameters from high-resolution luminosity measurements," J. Appl. Phys., vol. 71, pp. 4763-4770, 1992.

[23] S. Anders, A. Anders, and B. Jüttner, "Brightness distribution and current density of vacuum arc cathode spots," J. Phys. D: Appl. Phys., vol. 25, pp. 1591-1599, 1992.

[24] P. Siemroth, T. Schülke, and T. Witke, "Investigations of cathode spots and plasma formation of vacuum arcs by high speed microscopy and spectrography," IEEE Trans. Plasma Sci., vol. 25, pp. 571-579, 1997. 
[25] A. Anders, "Verfahren zur Beurteilung von Elektroden in Hochdruckentladungslampen. (Procedure of evaluating electrodes in high pressure discharge lamps)," German Democratic Republic Patent DD 279977 A1, 1990.

[26] T. Schülke and P. Siemroth, "Vacuum arcs cathode spots as a self-similarity phenomenon," IEEE Trans. Plasma Sci., vol. 24, pp. 63-64, 1996.

[27] J. E. Daalder, "Random walk of cathode arc spots in vacuum," J.Phys.D: Appl.Phys., vol. 16, pp. 1727, 1983.

[28] E. Hantzsche, B. Jüttner, and H. Pursch, "On the random walk of arc cathode spots in vacuum," J.Phys.D: Appl.Phys., vol. 16, pp. L173-L179, 1983.

[29] B. Jüttner, "The dynamics of arc cathode spots in vacuum," J. Phys. D: Appl. Phys., vol. 28, pp. 516522, 1995.

[30] B. Jüttner and I. Kleberg, "The retrograde motion of arc cathode spots in vacuum," J Phys. D: Appl. Phys., vol. 33, pp. 2025-2036, 2000.

[31] R. Tanberg, "Motion of an electric arc in a magnetic field under low gas pressure," Nature, vol. 124, pp. 371-372, 1929.

[32] S. Kajita, N. Ohno, Y. Tsuji, H. Tanaka, and S. Takamura, "Self-affine fractality of bifurcating arc trail in magnetized plasma," J. Phys. Soc. Jpn., vol. 79, p. 054501, 2010.

[33] V. F. Puchkarev and A. M. Murzakayev, "Current density and the cathode spot lifetime in a vacuum arc at threshold currents," J. Phys. D: Appl. Phys., vol. 23, pp. 26-35, 1990.

[34] P. E. Secker and I. A. George, "Preliminary measurements of arc cathode current density," J. Phys. D: Appl. Phys., vol. 2, pp. 918-920, 1969.

[35] B. Jüttner, H. Pursch, and S. Anders, "On the current density at the cathode of vacuum arcs," J. Phys D: Appl. Phys., vol. 17, pp. L111-L114, 1984.

[36] J. D. Cobine and C. J. Gallagher, "Current density of the arc cathode spot," Phys. Rev., vol. 74, pp. 1524-1530, 1948.

[37] E. Hantzsche and B. Jüttner, "Current density in arc spots," IEEE Trans. Plasma Sci., vol. 13, pp. 230-234, 1985.

[38] N. Vogel and B. Jüttner, "Measurements of the current density in arc cathode spots from the Zeeman splitting of emission lines," J. Phys. D: Appl. Phys., vol. 24, pp. 922-927, 1991.

[39] I. I. Beilis, "State of the theory of vacuum arcs," IEEE Trans. Plasma Sci., vol. 29, pp. 657-670, 2001.

[40] W. G. Chace and H. K. More, Exploding Wires. New York: Plenum Press, 1962.

[41] G. A. Mesyats, "Ecton mechanism of the vacuum arc cathode spot," IEEE Trans. Plasma Sci., vol. 23, pp. 879-883, 1995.

[42] G. A. Mesyats, Explosive Electron Emission. Ekaterinburg: URO Press, 1998.

[43] S. A. Barengolts, G. A. Mesyats, and D. L. Shmelev, "Structure and time behavior of vacuum arc cathode spots," IEEE Trans. Plasma Sci., vol. 31, pp. 809-816, 2003.

[44] G. A. Mesyats and S. A. Barengolts, "The cathode spot of a high-current vacuum arc as a multiecton phenomenon," IEEE Trans. Plasma Sci., vol. 29, pp. 704-707, 2001.

[45] E. Hantzsche, B. Jüttner, and G. Ziegenhagen, "Why vacuum arc cathode spots can appear larger than they are," IEEE Trans. Plasma Sci., vol. 23, pp. 55-64, 1995.

[46] A. Anders, S. Anders, B. Jüttner, W. Bötticher, H. Lück, and G. Schröder, "Pulsed dye laser diagnostics of vacuum arc cathode spots," IEEE Trans. Plasma Sci., vol. 20, pp. 466-472, 1992.

[47] A. Anders, S. Anders, B. Jüttner, and H. Lück, "High-resolution imaging of vacuum arc cathode spots," IEEE Trans. Plasma Sci., vol. 24, pp. 69-70, 1996.

[48] N. Vogel and V. A. Skvortsov, "Plasma parameters within the cathode spot of laser-induced vacuum arcs: Experimental and theoretical investigations," IEEE Trans. Plasma Sci., vol. 25, pp. 553-563, 1997.

[49] A. Batrakov, N. Vogel, S. E. Popov, D. Proskurovsky, D. Kudimov, and D. Nikitine, "Interferograms of a cathode spot plasma obtained with a picosecond laser," IEEE Trans. Plasma Sci., vol. 30, pp. 106-107, 2002. 
[50] A. Batrakov, S. Popov, N. Vogel, B. Jüttner, and D. I. Proskurovsky, "Plasma parameters of an arc cathode spot at the low-current vacuum discharge," IEEE Trans. Plasma Sci., vol. 31, pp. 817-826, 2003.

[51] I. G. Kesaev, Cathode Processes of an Electric Arc (in Russian). Moscow: Nauka, 1968.

[52] S. S. Arapov and N. B. Volkov, "The formation and structure of current cells in a vacuum arc cathode spot," Tech. Phys. Lett., vol. 29, pp. 1-4, 2003.

[53] B. Jüttner, "Cathode spots of electrical arcs (Topical Review)," J. Phys. D: Appl. Phys., vol. 34, pp. R103-R123, 2001.

[54] C. D. Child, "Discharge from hot CaO," Phys. Rev., vol. 32, pp. 492-511, 1911.

[55] R. C. Smith, R. D. Forrest, J. D. Carey, W. K. Hsu, and S. R. P. Silva, "Interpretation of enhancement factor in nonplanar field emitters," Appl. Phys. Lett., vol. 87, p. 013111, 2005.

[56] R. G. Forbes, C. J. Edgcombe, and U. Valdrè, "Some comments on models for field enhancement," Ultramicroscopy, vol. 95, pp. 57-65, 2003.

[57] R. Seelaboyina, J. Huang, J. Park, D. H. Kang, and W. B. Choi, "Multistage field enhancement of tungsten oxide nanowires and its field emission in various vacuum conditions," Nanotechnology, vol. 17, p. 4840, 2006.

[58] E. Hantzsche, "Arc spot ignition caused by sheath instability," IEEE Trans. Plasma Sci., vol. 25, pp. 527--532, 1997.

[59] A. E. Robson and P. C. Thonemann, "An arc maintained on an isolated metal plate exposed to a plasma," Proc. Phys. Soc., vol. 73, pp. 508-512, 1959.

[60] F. Schwirzke, "Unipolar arc model," J. Nucl. Mater., vol. 128-129, pp. 609-612, 1984.

[61] S. A. Barengolts, G. A. Mesyats, and M. M. Tsventoukh, "The ecton mechanism of unipolar arcing in magnetic confinement fusion devices," Nuclear Fusion, vol. 50, p. 125004, 2010.

[62] E. Hantzsche, "Unipolarbögen," Beitr. Plasmaphys., vol. 20, pp. 329-342, 1980.

[63] S. Kajita, S. Takamura, and N. Ohno, "Prompt ignition of a unipolar arc on helium irradiated tungsten," Nuclear Fusion, vol. 49, p. 032002, 2009.

[64] M. Tokitani and et al., "Exfoliation of the tungsten fibreform nanostructure by unipolar arcing in the LHD divertor plasma," Nuclear Fusion, vol. 51, p. 102001, 2011.

[65] S. Kajita, S. Takamura, and N. Ohno, "Motion of unipolar arc spots ignited on a nanostructured tungsten surface," Plasma Physics and Controlled Fusion, vol. 53, p. 074002, 2011.

[66] I. G. Brown, B. Feinberg, and J. E. Galvin, "Multiply stripped ion generation in the metal vapor vacuum arc," J. Appl. Phys., vol. 63, pp. 4889-4898, 1988.

[67] A. Anders, "Cohesive energy rule for vacuum arcs," in Emerging Applications of Vacuum-ArcProduced Plasma, Ion and Electron Beams, I. Brown and E. Oks, Eds. Dordrecht: Kluwer Academic Publishers, 2002, pp. 1-14.

[68] J. D. Cobine, "Effects of oxides and impurities on metallic arc reignition," Phys. Rev., vol. 53, p. 911, 1938.

[69] B. E. Djakov and R. Holmes, "Cathode spot structure and dynamcis in low-current vacuum arcs," $J$. Phys. D: Appl. Phys., vol. 7, pp. 569-580, 1974.

[70] B. Jüttner, "Erosion craters and arc cathode spots," Beitr. Plasmaphys., vol. 19, pp. 25-48, 1979.

[71] K. Jakubka and B. Jüttner, "The influence of surface conditions on the initiation, propagation and current density of unipolar arcs in fusion devices," J. Nucl. Mat., vol. 102, pp. 259-266, 1980.

[72] D. I. Proskurovsky and V. F. Puchkarev, "Formation of new emission centers on a cathode during current switching in vacuum. Formation of new emission centers," Sov.Phys.Tech.Phys., vol. 24, pp. 1474-1478, 1979.

[73] I. I. Beilis, "Vacuum arc cathode spot grouping and motion in magnetic fields," IEEE Trans. Plasma Sci., vol. 30, pp. 2124-2132, Dec 2002.

[74] G. Y. Yushkov and A. Anders, "Effect of the pulse repetition rate on the composition and ion chargestate distribution of pulsed vacuum arcs," IEEE Trans. Plasma Sci., vol. 26, pp. 220-226, 1998.

[75] J. Sasaki and I. G. Brown, "Ion spectra of vacuum arc plasma with compound and alloy cathodes," $J$. Appl. Phys., vol. 66, pp. 5198-5203, 1989. 
[76] J. Sasaki, K. Sugiyama, X. Yao, and I. Brown, "Multiple-species ion beams from titanium-hafnium alloy cathodes in vacuum arc plasmas," J. Appl. Phys., vol. 73, pp. 7184-7187, 1993.

[77] T. Schülke and A. Anders, "Ion charge state distributions of alloy-cathode vacuum arc plasmas," IEEE Trans. Plasma Sci., vol. 27, pp. 911-914, 1999.

[78] K. P. Savkin, Y. G. Yushkov, A. G. Nikolaev, E. M. Oks, and G. Y. Yushkov, "Generation of multicomponent ion beams by a vacuum arc ion source with compound cathode," Rev. Sci. Instrum., vol. 81, pp. 02A501-3, 2010.

[79] A. Anders, G. Yushkov, E. Oks, A. Nikolaev, and I. Brown, "Ion charge state distributions of pulsed vacuum arc plasmas in strong magnetic fields," Rev. Sci. Instrum., vol. 69, pp. 1332-1335, 1998.

[80] A. P. Ehiasarian, P. E. Hovsepian, R. New, and J. Valter, "Influence of steering magnetic field on the time-resolved plasma chemistry in cathodic arc discharges," J. Phys. D: Appl. Phys., vol. 37, pp. 2101-2106, 2004.

[81] I. A. Krinberg, "On the mechanism of the external magnetic field action on the electron temperature and ion charge state distribution in a vacuum arc plasma," Techn. Phys. Lett., vol. 29, pp. 504-506, 2003.

[82] E. Oks, I. G. Brown, M. R. Dickinson, R. A. MacGill, P. Spädtke, H. Emig, and B. H. Wolf, "Elevated ion charge states in vacuum arc plasmas in a magnetic field," Appl. Phys. Lett., vol. 67, pp. 200-202, 1995.

[83] E. M. Oks, A. Anders, I. G. Brown, M. R. Dickinson, and R. A. MacGill, "Ion charge state distributions in high current vacuum arc plasma in a magnetic field," IEEE Trans. Plasma Sci., vol. 24, pp. 1174-1183, 1996.

[84] E. Oks and A. Anders, "Measurements of the ion species of cathodic arc plasma in an axial magnetic field," IEEE Trans. Plasma Sci., vol. 39, pp. 1272-1276, 2011.

[85] F. J. Paoloni and I. G. Brown, "Some observations of the effect of magnetic field and arc current on the vacuum arc charge state distribution," Rev. Sci. Instrum., vol. 66, pp. 3855-3858, 1995.

[86] J. Rosén, A. Anders, and J. M. Schneider, "Plasma chemistry fluctuations in a reactive arc plasma in the presence of magnetic fields," Appl. Phys. Lett., vol. 80, pp. 4109-4111, 2002.

[87] J. Rosén, A. Anders, S. Mráz, and J. M. Schneider, "Charge-state-resolved ion energy distributions of aluminum vacuum arcs in the absence and presence of a magnetic field," J. Appl. Phys., vol. 97, pp. 103306-1-6, 2005.

[88] A. Anders, "Approaches to rid cathodic arc plasma of macro- and nanoparticles: a review," Surf. Coat. Technol., vol. 120-121, pp. 319-330, 1999.

[89] A. Anders, "Imaging the separation of cathodic arc plasma and macroparticles in curved magnetic filters," IEEE Trans. Plasma Sci., vol. 30, pp. 108-109, 2002.

[90] A. A. Plyutto, V. N. Ryzhkov, and A. T. Kapin, "High speed plasma streams in vacuum arcs," Sov. Phys. JETP, vol. 20, pp. 328-337, 1965.

[91] W. D. Davis and H. C. Miller, "Analysis of the electrode products emitted by dc arcs in a vacuum ambient," J. Appl. Phys., vol. 40, pp. 2212-2221, 1969.

[92] V. M. Lunev, V. G. Padalka, and V. M. Khoroshikh, "Plasma properties of a metal vacuum arc. II," Sov. Phys. Tech. Phys., vol. 22, pp. 858-861, 1977.

[93] J. E. Daalder, "Components of cathode erosion in vacuum arcs," J. Phys. D: Appl. Phys., vol. 9, pp. 2379-2395, 1976.

[94] I. G. Brown, P. Spädtke, D. M. Rück, and B. H. Wolf, "Beam intensity fluctuation characteristrics of the metal vapor vacuum arc ion source," Nucl. Instrum. Meth. Phys. Res. A, vol. 295, pp. 12-20, 1990.

[95] A. Anders, S. Anders, B. Jüttner, and I. G. Brown, "Time dependence of vacuum arc parameters," IEEE Trans. Plasma Sci., vol. PS-21, pp. 305-311, 1993.

[96] H. C. Miller, "Cathode ions from microsecond aluminum vacuum arcs," J. Appl. Phys., vol. 66, pp. 1107-1112, 1989.

[97] J. E. Galvin, I. G. Brown, and R. A. MacGill, "Charge state distribution studies of the metal vapor vacuum arc ion source," Rev. Sci. Instrum., vol. 61, pp. 583-585, 1990. 
[98] R. Sangines, A. M. Israel, I. S. Falconer, D. R. McKenzie, and M. M. M. Bilek, "Production of highly ionized species in high-current pulsed cathodic arcs," Appl. Phys. Lett., vol. 96, pp. 221501-3, 2010.

[99] A. Anders, "Time-dependence of ion charge state distributions of vacuum arcs: An interpretation involving atoms and charge exchange collisions," IEEE Trans. Plasma Sci., vol. 33, pp. 205-209, 2005.

[100] A. Anders, E. M. Oks, G. Y. Yushkov, K. P. Savkin, I. G. Brown, and A. G. Nikolaev, "Measurements of the total ion flux from vacuum arc cathode spots," IEEE Trans. Plasma Sci., vol. 33, pp. 1532-1536, 2005.

[101] A. Anders and G. Y. Yushkov, "Puzzling differences in bismuth and lead plasmas: evidence for the significant role of neutrals in cathodic vacuum arcs," Appl. Phys. Lett., vol. 91, p. 091502, 2007.

[102] A. Anders, E. M. Oks, and G. Y. Yushkov, "Production of neutrals and their effects on the ion charge states in cathodic vacuum arc plasmas," J. Appl. Phys., vol. 102, p. 043303, 2007.

[103] J. Rosén, A. Anders, L. Hultman, and J. M. Schneider, "Charge state and time resolved plasma composition of a pulsed zirconium arc in a nitrogen environment," J. Appl. Phys., vol. 96, pp. 47934799, 2004.

[104] J. M. Schneider, A. Anders, I. G. Brown, B. Hjörvarsson, and L. Hultman, "Temporal development of the plasma composition of a pulsed aluminum plasma stream in the presence of oxygen," Appl. Phys. Lett., vol. 75, pp. 612-614, 1999.

[105] J. E. Daalder, "Erosion and the origin of charged and neutral species in vacuum arcs," J. Phys. D: Appl. Phys., vol. 8, pp. 1647-1659, 1975.

[106] R. N. Tarrant, M. M. M. Bilek, J. Pigott, and D. R. McKenzie, "Plasma transport and optical flares in high-density plasmas produced from a pulsed cathodic arc," Surf. Coat. Technol., vol. 186, pp. 10-16, 2004.

[107] P. A. Ni and A. Anders, "Supersonic metal plasma impact on a surface: an optical investigation of the pre-surface region," J. Phys. D: Appl. Phys., vol. 43, p. 135201, 2010.

[108] H. Wu and A. Anders, "Energetic deposition of metal ions: Observation of self-sputtering and limited sticking for off-normal angles of incidence," J. Phys D: Appl. Phys., vol. 43, p. 065206, 2010.

[109] S. A. Popov, A. V. Batrakov, R. Methling, D. Uhrlandt, and K.-D. Weltmann, "Spectroscopic study of a single vacuum-arc cathode spot," IEEE Trans. Plasma Sci., vol. 37, pp. 1419-1425, 2009.

[110] I. I. Aksenov, P. Antuf'ev Yu, V. G. Bren, V. G. Padalka, A. I. Popov, and V. M. Khoroshikh, "Effect of electron magnetization in vacuum-arc plasma on the kinetics of the synthesis of nitrogencontaining coatings," Sov. Phys -Tech. Phys., vol. 26, pp. 184-187, 1981.

[111] B. F. Coll, R. Fontana, A. Gates, and P. Sathrum, "(Ti-Al)N advanced films prepared by arc process," Mater. Sci. Eng. A, vol. 140, pp. 816-824, 1991.

[112] V. N. Zhitomirsky, U. Kinrot, B. Alterkop, R. L. Boxman, and S. Goldsmith, "Influence of gas pressure on the ion current and its distribution in a filtered vacuum arc deposition system," Surf. Coat. Technol., vol. 87-8, pp. 263-270, 1996.

[113] J. Sjolen, L. Karlsson, S. Braun, R. Murdey, A. Horling, and L. Hultman, "Structure and mechanical properties of arc evaporated Ti-Al-O-N thin films," Surf. Coat. Technol., vol. 201, pp. 6392-6403, 2007.

[114] M. K. Puchert, C. A. Davis, D. R. McKenzie, and B. W. James, "Gas-plasma interactions in a filtered cathodic arc," J. Vac. Sci. Technol. A, vol. 10, pp. 3493-3498, 1992.

[115] P. Spädtke, H. Emig, B. H. Wolf, and E. Oks, "Influence of gas added to the MEVVA discharge on the extracted ion beam," Rev. Sci. Instrum., vol. 65, pp. 3113-3118, 1994.

[116] A. G. Nikolaev, E. M. Oks, and G. Y. Yushkov, "Effect of residual gas on the ion charge distribution in vacuum arc discharge plasmas," Zhurnal Tekhnicheskoi Fiziki, vol. 68, pp. 24-28, 1998.

[117] H. Kelly, A. Lepone, and A. Marquez, "Characterization of plasma-gas interactions in a copper cathodic arc operated in oxygen," J. Appl. Phys., vol. 89, pp. 1567-1572, 2001. 
[118] A. Lepone, H. Kelly, and A. Marquez, "Role of metallic neutrals and gaseous molecular ions in a copper cathodic arc operated with oxygen gas," J. Appl. Phys., vol. 90, pp. 3174-3181, 2001.

[119] C. R. Negus and N. J. Peacock, "Local regions of high-pressure plasma in a vacuum spark," $J$. Phys. D: Appl. Phys., vol. 12, pp. 91-111, 1979.

[120] R. Beier and H.-J. Kunze, "Observation of line radiation from highly charged Mo ions in a vacuum spark plasma," Z. Physik A, vol. 285, pp. 347-352, 1978.

[121] M. Hebach and et al., "Time Evolution of the X-ray Emission from a Micropinch in a Vacuum Spark Discharge," Europhys. Lett., vol. 21, p. 311, 1993.

[122] A. A. Plyutto, K. N. Kervalidze, and I. F. Kvartskhava, "Spark source for multiply-charged ions," Atomic Energy, vol. 3, pp. 925-928, 1957.

[123] G. Y. Yushkov and A. Anders, "Extractable, elevated ion charge states in the transition regime from vacuum sparks to high current vacuum arcs," Appl. Phys. Lett., vol. 92, p. 041502, 2008.

[124] G. Y. Yushkov and A. Anders, "Physical limits for high ion charge states in pulsed discharges in vacuum," J. Appl. Phys., vol. 105, pp. 043303-5, 2009.

[125] A. Anders, "A periodic table of ion charge-state distributions observed in the transition region between vacuum sparks and vacuum arcs," IEEE Trans. Plasma Sci., vol. 29, pp. 393-398, 2001.

[126] B. M. Johnson, A. Hershcovitch, I. G. Brown, F. Liu, and A. Anders, "Results from z-Mevva: A new source for heavy-ion accelerators," in IEEE Particle Accelerator Conference, Vancouver, B.C., 1997.

[127] A. Hershcovitch, B. M. Johnson, F. Liu, A. Anders, and I. G. Brown, "Results from energetic electron beam metal vapor vacuum arc and z-discharge plasma metal vapor arc: Development of new sources of intense high charge state heavy-ion beams," Rev. Sci. Instrum., vol. 69, pp. 798-800, 1998.

[128] N. Debolt, A. Hershcovitch, B. M. Johnson, N. Rostoker, A. VanDrie, and F. Wessel, "Recent results from the low inductance Z-discharge metal vapor ion source," Rev. Sci. Instrum., vol. 73, pp. 741-743, 2002.

[129] A. S. Bugaev, V. I. Gushenets, G. Y. Yushkov, E. M. Oks, A. Anders, I. Brown, A. Hershkovitch, and P. Spaedtke, "Generation of multiply charged ions in vacuum arc discharges (in Russian)," Izv. Vys. Ucheb. Zav. (Fizika), vol. 44, pp. 15-22, 2001.

[130] A. Bugaev, V. Gushenets, G. Yushkov, E. Oks, T. Kulevoy, A. Hershcovitch, and B. M. Johnson, "Electron-beam enhancement of ion charge state fractions in the metal-vapor vacuum-arc ion source," Appl. Phys. Lett., vol. 79, pp. 919-921, 2001.

[131] V. A. Batalin, A. S. Bugaev, V. I. Gushenets, A. Hershcovitch, B. M. Johnson, A. A. Kolomiets, R. P. Kuibeda, T. V. Kulevoy, E. M. Oks, V. I. Pershin, S. V. Petrenko, D. N. Seleznev, and G. Y. Yushkov, "Electron-beam enhancement of the metal vapor vacuum arc ion source," J. Appl. Phys., vol. 92, pp. 2884-2889, 2002.

[132] A. V. Vodopyanov, S. V. Golubev, V. G. Zorin, S. V. Razin, A. V. Vizir, A. G. Nikolaev, E. M. Oks, and G. Y. Yushkov, "Multiple ionization of metal ions by ECR heating of electrons in vacuum arc plasmas," Rev. Sci. Instrum., vol. 75, pp. 1888-1890, 2004.

[133] A. Vodopyanov, S. Golubev, D. Mansfeld, A. Nikolaev, E. Oks, S. Razin, K. Savkin, and G. Yushkov, "Generation of multiply charged refractory metals in an electron-cyclotron resonant discharge in a direct magnetic trap," Techn .Phys., vol. 50, pp. 1207-1211, 2005.

[134] A. Vodopyanov, S. Golubev, D. Mansfeld, A. Nikolaev, E. Oks, V. Khizhnyak, and G. Yushkov, "Multiple ionization of vacuum-arc-generated metal ions in a magnetic trap heated by high-power microwave radiation," Techn. Phys. Lett., vol. 33, pp. 872-874, 2007.

[135] G. Y. Yushkov, E. M. Oks, A. Anders, and I. G. Brown, "Effect of multiple current spikes on the enhancement of ion charges states of vacuum arc plasmas," J. Appl. Phys., vol. 87, pp. 8345-8350, 2000.

[136] G. Y. Yushkov, A. Anders, E. M. Oks, and I. G. Brown, "Ion velocities in vacuum arc plasmas," J. Appl. Phys., vol. 88, pp. 5618-5622, 2000.

[137] E. Byon and A. Anders, "Ion energy distribution functions of vacuum arc plasmas," J. Appl. Phys., vol. 93, pp. 1899-1906, 2003. 
[138] M. F. Artamonov, V. I. Krasov, and V. L. Paperny, "Generation of multiply charged ions from a cathode jet of a low-energy vacuum spark," J. Phys. D: Appl. Phys., vol. 34, pp. 3364-3367, 2001.

[139] S. P. Gorbunov, V. P. Krasov, V. L. Paperny, and A. S. Savyelov, "Flow of multiple charged accelerated metal ions from low-inductance vacuum spark," J. Phys. D: Appl. Phys., vol. 39, pp. 5002-5007, 2006.

[140] A. Anders, I. G. Brown, M. R. Dickinson, and R. A. MacGill, "High energy metal ion implantation using a novel, broad beam, Marx-generator-based ion source "Magis"," Nucl. Instrum. Meth. Phys. Res. B, vol. 127/128, pp. 992-995, 1997.

[141] A. Anders, I. G. Brown, R. A. MacGill, and M. R. Dickinson, "Vacuum-spark metal ion source based on a modified Marx generator," IEEE Trans. Plasma Sci., vol. 25, pp. 718-721, 1997. 